\title{
USING A RUNTIME MEASUREMENT DEVICE WITH MEASUREMENT-BASED WCET ANALYSIS *
}

\author{
Bernhard Rieder, Ingomar Wenzel, Klaus Steinhammer, and Peter Puschner \\ Institut für Technische Informatik \\ Technische Universität Wien \\ Treitlstraße 3/182/1 \\ 1040 Wien, Austria \\ bernhard@vmars.tuwien.ac.at
}

\begin{abstract}
:
The tough competition among automotive companies creates a high cost pressure on the OEMs. Combined with shorter innovation cycles, testing new safety-critical functions becomes an increasingly difficult issue [4]. In the automotive industry about $55 \%$ of breakdowns can be traced back to problems in electronic systems. About $30 \%$ of these incidents are estimated to be caused by timing problems [7]. It is necessary to develop new approaches for testing the timing behavior on embedded and real-time systems.

This work describes the integration of runtime measurements using an external measurement device into a framework for measurement-based worst-case execution time calculations. We show that especially for small platforms using an external measurement device is a reasonable way to perform execution time measurements. Such platforms can be identified by the lack of a time source, limited memory, and the lack of an external interface. The presented device uses two pins on the target to perform run-time measurements. It works cycle accurate for frequencies up to $200 \mathrm{MHz}$, which should be sufficient for most embedded devices.
\end{abstract}

\section{INTRODUCTION}

Over the last years more and more automotive subsystems have been replaced by electronic control units (ECUs) which are interconnected by high

\footnotetext{
* This work has been supported by the FIT-IT research projects "Automatic Test Data Generation for WCET Measurements (ATDGEN)" and "Model-based Development of distributed Embedded Control Systems (MoDECS-d)".
} 
dependable bus systems like FlexRay, TTP/C or CAN. Much effort has been put into increasing the reliability of communication and scheduling and great advances in these areas have been made. However, timing analysis, especially worst-case Execution Time (WCET) analysis of automotive applications, is still a challenge. This is mainly due to two factors which cumulatively increase complexity of timing analysis: More and more functionality is integrated within single ECUs [4] and the architecture of the processors is getting more complex, especially by features such as caches, pipelining, branch prediction, out of order execution and others [3]. Additionally, processor vendors do not offer accurate data sheets describing the features of their processors, so that those are unknown or have to be figured out by reverse engineering [8]. Without detailed knowledge about processor internals static timing analysis, that is calculating the time required for the execution of code without actually executing it, is often not possible.

Therefore, novel approaches use a combination of static analysis and execution time measurements to calculate a WCET bound [10]. The proposed method consists of static analysis, control flow graph decomposition, test data generation, execution-time measurement and the final calculation step. All steps are performed automatically without user interaction. The method is described in Section 3.

Computing resources of embedded applications are often limited and therefore not suitable for runtime measurements. Typical limitations are the lack of a time source, limited memory (no location to store measurement data), and the lack of an external interface (no way to transfer measurement data to host). As a solution we developed an external measurement device, which is based on an FPGA and therefore very flexible and inexpensive. We demonstrate the usage of the execution time measurement device by performing WCET calculations using a HSC12 microcontroller evaluation board. The proposed solution uses only a single instruction per measurement point and two pins for the interface to the measurement device.

\section{Structure of this Article}

This paper is structured as follows: In Section 2 we present related work in the domain of dynamic WCET estimation and execution time measurement. The measurement-based WCET analysis approach is described in Section 3. Section 4 outlines basic requirements when performing execution time measurements for timing analysis. In Section 5 we describe the hardware and firmware design of the measurement device. Section 6 explains how execution time measurements are performed by the timing analysis framework. The conducted experiments are described in Section 7. At last, Section 8 gives a short conclusion and an overview of open topics. 


\section{Contributions}

The first contribution is the introduction of a dedicated runtime measurement device (RMD). The device works for CPU frequencies of up to $200 \mathrm{MHz}$. It is linked to the target using a simple 2-wire connection. Since the device is built using a field programmable gate array (FPGA) it can easily be extended or reconfigured and is very flexible. The device is especially useful to perform execution time measurements on targets with limited resources.

Second, the device is seamlessly integrated into an novel developed measurement-based WCET calculation framework as described in [10]. All operations of the measurement-based WCET analysis are performed fully automatic without user interaction.

\section{RELATED WORK}

Petters [5] describes a method to split an application down to "measurement blocks" and to enforce execution paths by replacing conditional jumps either by NOPs or unconditional jumps, eliminating the need for test data. The drawback is that the application is partitioned manually and that the measured, semantically changed application may differ from the real application.

Bernat et al. introduce the term of a "probabilistic hard real-time system". They combine a segmented measurement approach and static analysis, however they use test data supplied by the user. Therefore they cannot guarantee a WCET bound but only give a probabilistic estimation, based on the used test data [1].

Petters describes various ways to collect execution traces of applications in [6]. He outlines various ways how to place "observation points" and discusses benefits and drawbacks of the presented methods.

\section{MEASUREMENT-BASED WCET ANALYSIS}

The proposed measurement-based timing analysis (MBTA) is performed in five steps [9] as shown in Figure 1

The individual steps are explicitly described below. The measurement device hardware is used in step (4) but the description of the other steps is necessary to understand how the MBTA approach works. It is also important to mention that the current implementation is limited to acyclic code (code without loops). Since most modeling tools typically produce acyclic code this does not strongly limit the applicability of the presented method.

\section{Static Program Analysis (1)}

This step is used to extract structural and semantic information from the $\mathrm{C}$ source code. The information is needed to perform the next steps. 


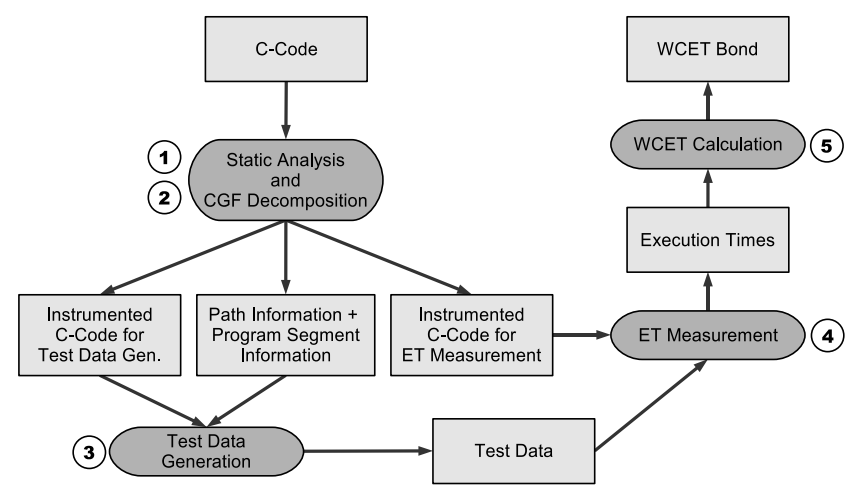

Figure 1. MBTA Framework Overview

\section{Control Flow Graph Decomposition (2)}

During this phase, the control flow graph $C F G$ of the analyzed program is split up into smaller program segments $P S$. The subdivision is controlled by the number of execution paths which remain within each PS, a number which is denoted as path bound. The command line argument for the path bound is adjustable from 1 to $2^{32}$ and controls the correlation between calculation time and the number of required measurements: A high path bound causes a lower number of PS and less measurement points but the sum of execution paths through all PS increases. Since each execution path of each PS has to be measured, a test data set is required for each path. Since test data calculation is very time consuming, the calculation time rises significantly [11].

\section{Test Data Generation (3)}

In this step test data is calculated to execute all execution paths of each PS. The test data is acquired using a multi-stage process: Random search which is very fast is used to find most data sets. Where random search fails, model checking is used. Model checking is exhaustive, that means if there exists a data set to execute a certain path, then it will be found. If no data can be found for a particular execution path then the path is semantically infeasible. An example for semantically infeasible paths are mutual exclusive conditions in consecutive if statements. We used the CBMC model checker [2], which is a very fast bounded model checker that can directly process $C$ input.

\section{Execution Time Measurements (4)}

Using the generated test data all feasible paths within each PS are executed. The result of this step is an execution time profile for each PS which includes 
the execution time of each path. The WCET of a given PS is the maximum of all execution time values.

\section{WCET Calculation (5)}

The last step is the calculation of a WCET bound. The current implementation of the WCET calculation tool described in [10] uses a longest path search algorithm for a directed, acyclic graph which a single start and ending node to compute a WCET bound over all PS. This can lead to overestimations under certain circumstances, namely when the execution path with the WCET of $P S_{x}$ inhibits the execution of the path featuring the WCET of $P S_{y}$. In this case WCET $\left(P S_{x}\right)$ and WCET $\left(P S_{y}\right)$ are accumulated in the WCET of the whole application leading to overestimation. This effect can be reduced by increasing the path bound.

\section{PERFORMING EXECUTION TIME MEASUREMENTS}

Execution time measurements are a precondition for the described timing analysis method. There are various methods to place instrumentation points and to perform measurements [6].

As a first method, execution traces can be made, using a cycle accurate simulator. In most cases this is not possible due to missing CPU models and documentation.

Second, pure software instrumentation can be used: A few instructions are inserted into the application that read the value of an internal time source, commonly a cycle counter located in the CPU, and write it to a output port or to a memory location. The drawback of this method is that several instructions are needed and therefore the code size and the execution time can be considerably increased.

The third option is to use pure hardware instrumentation. A logic analyzer is connected to the address bus and records the access to the memory. The advantage of this method is that no alterations on the applications are necessary. The drawback of this method is that it is expensive (the logic analyzer) and that the connection to the address bus is not always possible or the CPU has an internal instruction cache.

An interesting alternative is to use software supported hardware instrumentation. We selected this option because the modifications on the software are very lightweight (a single assembler instruction is sufficient) and the resource and execution time consumption on the target hardware is small. Since logic analyzers are often difficult to control from within an application, expensive and oversized for this task we decided to design a custom device to perform execution time measurements. 


\section{RUNTIME MEASUREMENT DEVICE (RMD)}

The Runtime Measurement Device (RMD) acts as interface between the target and the host. It collects timestamps issued from the target and transfers them to the host. The timestamps are internally generated.

The RMD consists of a custom designed FPGA board with an Altera ${ }^{\circledR}$ Cyclone $^{\mathrm{TM}}$ EP1C12C6 device which is additionally equipped with $64 \mathrm{k}$ of external memory (for the CPU core) and interface circuits for USB, Ethernet, RS232, RS485, ISO-K, and CAN. A modern FPGA evaluation board can also be used instead, however with a total price of approximately 300.00 Euros (with USB interface only) the custom made board is cheaper than most evaluation boards. The firmware is split up in two parts. The first part runs on a NIOS ${ }^{\circledR}$ CPU core which is clocked with $50 \mathrm{MHz}$ and controls the communication with the host computer. The second part is written in VHDL (Very High Speed Integrated Circuit Hardware Description Language) and operates at a clock frequency of $200 \mathrm{MHz}$. This part contains the time base, which is a 32 bit counter, a FIFO to store up to 128 measurement values until they are transfered to the host and additional glue logic which recognizes measurement points and stores the actual counter value in the FIFO and synchronizes communication with the CPU core.

Since most of the design is implemented within the FPGA firmware the whole method is very flexible and can easily be adopted for custom application needs. Changes in the configuration can simply be made by uploading a new firmware design on the FPGA.

\section{Operation}

The measurement device is designed to work in two different modes. The first mode, the 2-wire mode, uses two dedicated IO pins for the measurements as depicted in Figure 1. Measurement starts when one signal drops to low. The internal counter is released and starts counting. On each measurement point, one signal drops to low, causing the counter value to be stored in the FIFO, and the other signal rises to high. If both signals are low for a adjustable amount of time, the measurement stops. According to the FIFO size up to 128 measurement points can be recorded on a single run.

The second mode is the analyzer mode. This mode is designed for very small devices. In this mode the measurement device is connected to the CPU address bus and records the time at certain predefined locations. The addresses where time tamps have to be recorded are stored in a one bit wide RAM. Measurements are taken when the output of the RAM is logical "1". The advantage of this mode is that there need be no alterations on the target code. The disadvantages are that knowledge about the physical location of the measurement 


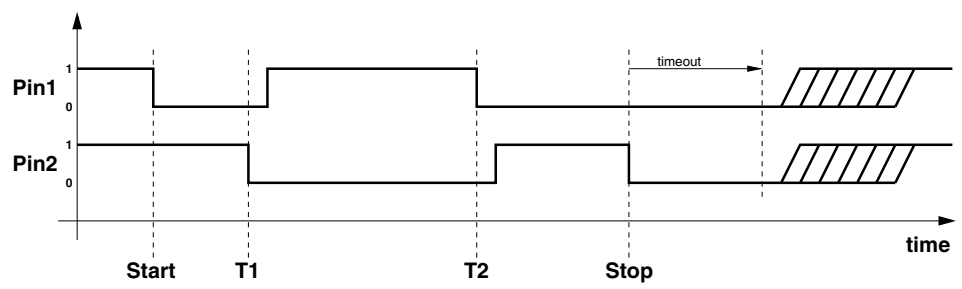

Figure 1. 2-Wire Interface Signal Waveform

points is necessary and that physical access to the address bus of the device is required, so it cannot be used for devices with on-board E(E)PROM storage.

\section{INTEGRATION IN THE ANALYSIS FRAMEWORK}

The measurement framework consists of a set of virtual base classes shown in Figure 2. For each virtual base class a non-virtual subclass is loaded at runtime, according to the underlying hardware.

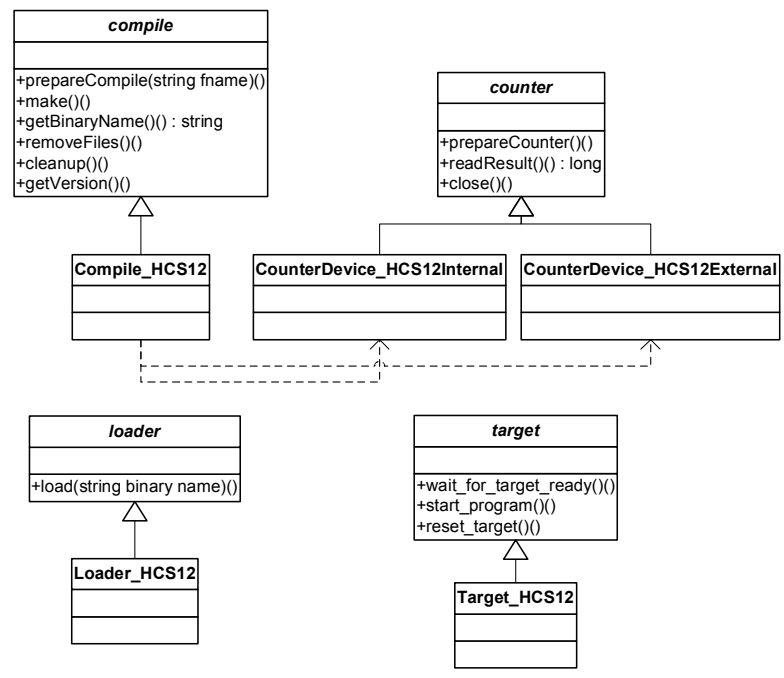

Figure 2. Measurement Application Class Framework [9]

The compile class is used to compile the application on the host and to generate a stub for the target to handle the communication with the host, load the test data, and to execute the application. The counter class activates the source code for starting and stopping the counter on the target and handles the communication between the host and the counting device for both, internal and external counting devices. The loader class is used to upload the application 
binary onto the target, and the target class handles the communication between target and host from the host side.

The proposed design makes the execution time measurement application very flexible and allows an easy adoption for new target hardware. Since the measurement runs are all performed in the same way, using only a different set of subclasses, the whole framework can be adopted to support new target platforms by implementing additional subclasses.

\section{EXPERIMENTAL RESULTS}

We performed a series of tests using the measurement-based WCET analysis framework in combination with the internal counter device of the HCS12 microcontroller (HCS12_INTERNAL) and the runtime measurement device (HCS12_EXTERNAL). As expected, we got slightly bigger values using HCS12_INTERNAL during first test runs. This is caused by the different instrumentation methods (using the internal counter requires more instructions and therefore takes longer) and can be eliminated through calibration. To calibrate, a series of zero-length code sequences are measured and the result is subtracted from the actual measurements. For all tests the resulting WCET values were the same using both measurement methods.

The test cases we used were developed from a simple example from our regression tests (nice_partitioning_semantisch_richtig) and from automotive applications (ADCKonv, AktuatorSysCtrl, and AktuatorMotorregler). Figure 3 [9] shows the test results for all case studies for different "Path Bounds (b)". As mentioned before, $b$ controls the maximum number of syntactically possible paths within a Program Segment (PS) after the CFG decomposition step. "Program Segments (PS)" shows in how many segments the Application was broken down. The next column "Paths After Dec.(2)" represents the sum of all syntactically possible paths through each PSs of the application. Interesting values are at the first and on the last line of each test case. When $b$ equals 1 the total number of paths after the decomposition step equals the number of basic blocks, since one basic block comprises exactly one path. In the last line there is only a single PS and therefore the number of paths after decomposition equals the number of syntactically possible paths of the whole application.

"Paths Heuristic" and "Paths MC" describe how many paths were covered by random test data generation and how many by model checking. "Infeasible Paths" denotes the number of infeasible paths that were discovered during model checking. Note that these paths are also included in the number of paths covered by model checking. Infeasible paths are paths that are syntactically possible but can never be executed because the semantics of the code does not allow so. Since the number of paths covered by model checking is similar to 


\begin{tabular}{|c|c|c|c|c|c|c|c|c|c|c|c|c|c|}
\hline Application Name & 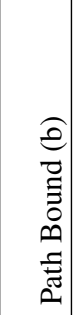 & 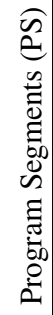 & 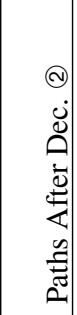 & 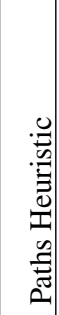 & 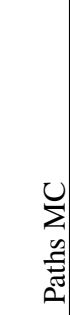 & 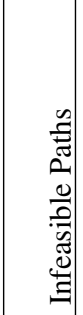 & 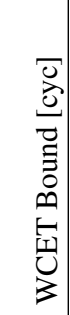 & $\begin{array}{c}\bar{n} \\
\tilde{U} \\
\sum_{0} \\
\tilde{\Xi} \\
\Xi\end{array}$ & 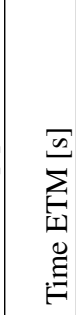 & 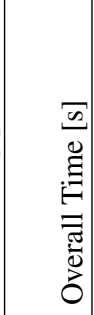 & 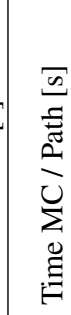 & 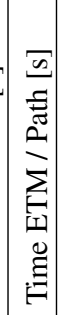 & ص2 \\
\hline \multirow{5}{*}{$\begin{array}{l}\text { nice_partitioning } \\
\text { semantisch_richtig } \\
(46 \text { LOC) }\end{array}$} & 1 & 30 & 30 & 6 & 24 & 0 & 151 & 34 & 175 & 209 & 1.42 & 5.8 & 1.0 \\
\hline & 5 & 6 & 14 & 4 & 10 & 0 & 151 & 15 & 39 & 54 & 1.50 & 2.8 & 2.3 \\
\hline & 10 & 3 & 14 & 3 & 11 & 0 & 151 & 16 & 21 & 37 & 1.45 & 1.5 & 4.7 \\
\hline & 20 & 2 & 18 & 2 & 16 & 3 & 150 & 22 & 16 & 38 & 1.38 & 1.1 & 9.0 \\
\hline & 100 & 1 & 72 & 1 & 71 & 46 & 129 & 106 & 12 & 118 & 1.49 & 0.5 & 72.0 \\
\hline \multirow{4}{*}{$\begin{array}{l}\text { ADCKonv } \\
\text { (321 LOC) }\end{array}$} & 1 & 31 & 31 & 31 & 0 & 0 & 872 & 24 & 192 & 216 & n.a. & 6.2 & 1.0 \\
\hline & 10 & 3 & 17 & 8 & 9 & 8 & 870 & 31 & 22 & 53 & 3.44 & 2.4 & 5.7 \\
\hline & 100 & 2 & 74 & 8 & 66 & 60 & 872 & 220 & 17 & 237 & 3.33 & 1.2 & 37.0 \\
\hline & 000 & 1 & 144 & 12 & 132 & 132 & 872 & 483 & 11 & 494 & 3.66 & 0.9 & 144.0 \\
\hline \multirow{3}{*}{$\begin{array}{l}\text { AktuatorSysCtrl } \\
\text { (274 LOC) }\end{array}$} & 1 & 54 & 54 & 54 & 0 & 0 & 173 & 26 & 318 & 344 & n.a. & 5.9 & 1.0 \\
\hline & 10 & 14 & 36 & 36 & 0 & 0 & 173 & 10 & 85 & 95 & n.a. & 2.4 & 2.6 \\
\hline & 100 & 1 & 97 & 18 & 79 & 72 & 131 & 191 & 10 & 201 & 2.42 & 0.4 & 97.0 \\
\hline \multirow{4}{*}{$\begin{array}{l}\text { AktuatorMotorre- } \\
\text { gler }(1150 \\
\text { LOC) }\end{array}$} & 1 & 171 & 171 & 165 & 6 & 6 & n.a. & 468 & 1289 & 1757 & 78.0 & 7.8 & 1.0 \\
\hline & 10 & 14 & 92 & 63 & 29 & 23 & 3445 & 841 & 116 & 957 & 29.0 & 1.7 & 6.6 \\
\hline & 100 & 7 & 336 & 57 & 279 & 247 & 3323 & 7732 & 62 & 7794 & 27.7 & 0.7 & 48.0 \\
\hline & 1000 & & 1455 & 82 & 1373 & 1325 & 3298 & 41353 & 49 & 41402 & 30.1 & & 291.0 \\
\hline
\end{tabular}

Figure 3. Test Results Of Case Studies

the number of infeasible paths (except for the first example) we see that most of the feasible paths could be found by random test data generation.

"WCET Bound [cyc]" gives the estimated WCET in processor cycles. To identify performance bottlenecks we did not only measure the time required for the complete WCET estimation ("Overall Time") but also how much of this time budget was consumed by the analysis ("Time MC"), which consists mainly of model checking but also includes static analysis, CFG decomposition and random test data generation, and how much time was consumed by execution time measurements ("Time ETM"), which consists of code generation, compilation and uploading the binary to the target.

We also observed the performance of our solution relative to the number

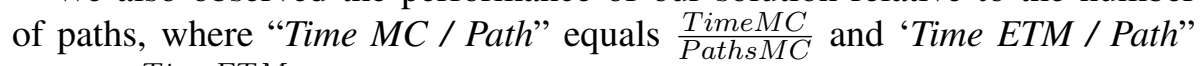
equals $\frac{\text { TimeETM }}{\text { feasiblePaths }}$. While the time required for model checking a single path within a PS is approximately constant for each test case, the time required for the execution time measurement of an individual path drops with the number of program segments. This is due to the fact that the current implementation is very simple and measures only a single PS at a time. To measure another PS the application needs to be recompiled and uploaded to the target again. On bigger 
applications higher values for $b$ should be used so the time fraction required for recompilation and uploading is much less than for a given examples. The last column ("Paths / PS") shows the average number of syntactically possible paths through a PS.

Regarding the path bound $b$ it can also be noted that the quality of the estimated WCET bound improves with a rising path bound. This is caused by the fact that semantically infeasible paths are only detected by the model checker when they are located within the same PS. Therefore, the bigger the PSs the more infeasible paths can be eliminated.

The fact that the longest measurement runs took little more than 11 hours (for current industrial code) is very promising. Generating test data sets and performing the necessary measurements manually for an application of this size would take a few days at least. We think that this approach can significantly improve the testing process. It should also be noted that test data are stored and reused for later runs. For applications featuring many infeasible paths we are currently working on a solution to identify groups of infeasible paths in a single run of the model checker.

With the case study "AktuatorMotorregler" we reached the computational limit of the model checker when we set $b$ to 1 therefore we could not get an WCET estimation value in this case.

\section{CONCLUSION AND FURTHER WORK}

We found that the measurement-based WCET analysis approach in combination with the presented device gives the application developer a powerful tool to perform WCET analysis. The most powerful feature is that everything is performed automatically. This saves valuable working time and eliminates cumbersome tasks like test data generation and performing manual measurements. The newly introduced measurement device makes it possible to perform run time measurements on small devices which normally would lack the appropriate hardware support (time source, memory or interface). The measurement device is cheap - and since it is based on a programmable logic device - very flexible, which allows the adaption for other hardware devices if necessary.

An important drawback is that, depending on the used RMD-target interface, at least two signal pins are required to perform measurements. Therefore the measurement device cannot be used when less than two pins are free.

The next step is to overcome limitations of the current measurement framework: loops and functions are not supported in the prototype. We are confident that these are only limitations of the current design of the prototype and of the measurement method itself. Currently a new version of the prototype which supports loops and function calls is in development. 
The test data generation by model checking can be boosted by cutting off infeasible subtrees in the dominator tree. If a node cannot be reached then all other nodes dominated by it are unreachable as well. However the current implementation makes no use of this information and checks each leaf in the dominator tree which represents an unique path within a program segment.

An interesting area for improvement is the reconfiguration the RMD firmware from within the framework for different types of target hardware.

Additionally we are working on a solution to make the measurement-based approach work on more complex architectures, as those are increasingly used in new embedded solutions. The presence of caches, pipelines and out-of-order execution units impose an internal state on the processor. Different hardware states at the same instruction can result in different execution times. Therefore we are searching ways to impose a well defined hardware state while loosing a minimum on performance.

\section{REFERENCES}

[1] G. Bernat, A. Colin, and S. M. Petters. WCET Analysis of Probabilistic Hard Real-Time Systems. RTSS, 00:279, 2002.

[2] E. Clarke and D. Kroening. ANSI-C Bounded Model Checker User Manual. August 2 2006.

[3] R. Heckmann, M. Langenbach, S. Thesing, and R. Wilhelm. The influence of processor architecture on the design and the results of WCET tools. Proceedings of the IEEE, 91(7):1038-1054, 2003.

[4] H. Heinecke, K. Schnelle, H. Fennel, J. Bortolazzi, L. Lundh, J. Leflour, J. Maté, K. Nishikawa, and T. Scharnhorst. AUTomotive Open System ARchitecture-An IndustryWide Initiative to Manage the Complexity of Emerging Automotive E/E Architectures. Proc. Convergence, SAE-2004-21-0042, 2004.

[5] S. Petters. Bounding the execution time of real-time tasks on modern processors. RealTime Computing Systems and Applications, 2000. Proceedings. Seventh International Conference on, pages 498-502, 2000.

[6] S. M. Petters. Comparison of trace generation methods for measurement based WCET analysis. In 3nd Intl. Workshop on Worst Case Execution Time Analysis, Porto, Portugal, July 1 2003. Satellite Workshop of the 15th Euromicro Conference on Real-Time Systems.

[7] Rapita Systems. Rapitime whitepaper. 2005.

[8] C. Thomborson and Y. Yu. MEASURING DATA CACHE AND TLB PARAMETERS UNDER LINUX. Proceedings of the 2000 Symposium on Performance Evaluation of Computer and Telecommunication Systems, pages 383-390, 2000.

[9] I. Wenzel. Measurement-Based Timing Analysis of Superscalar Processors. PhD thesis, Technische Universität Wien, Institut für Technische Informatik, Treitlstr. 3/3/182-1, 1040 Vienna, Austria, 2006.

[10] I. Wenzel, R. Kirner, B. Rieder, and P. Puschner. Measurement-based worst-case execution time analysis. Software Technologies for Future Embedded and Ubiquitous Systems, 2005. SEUS 2005. Third IEEE Workshop on, pages 7-10, 2005. 
[11] I. Wenzel, B. Rieder, R. Kirner, and P. Puschner. Automatic timing model generation by cfg partitioning and model checking. In Proc. Conference on Design, Automation, and Test in Europe, Mar. 2005. 